\title{
Nefropatía lúpica
}

\author{
Luis Fernando Pinto Peñaranda ${ }^{1}$ \\ 1 Medicina Interna - Reumatología, Especialidades Médicas - Unidad de Investigaciones, \\ Hospital Pablo Tobón Uribe, Medellín - Colombia
}

\begin{abstract}
Resumen
La nefritis lúpica (NL) se presenta entre el 30 y $70 \%$ de los pacientes con lupus eritematoso sistémico (LES), dependiendo de la raza y el sexo, ocurre temprano en la enfermedad y predominan las formas graves, clases III, IV y mixtas (V + III o V + IV). El 50\% de los adultos y 70\% de los niños colombianos con lupus sufren NL en algún momento de la vida; en esta población el $25 \%$ de los niños y el $38 \%$ de los adultos presentan síndrome nefrótico, la tasa de remisión a 6 meses es baja, la proteinuria en rango nefrótico y la elevación de creatinina basal, predicen falla en el logro de remisión a 6 meses.
\end{abstract}

Palabras clave: Lupus, Nefritis, Síndrome Nefrótico, Glomerulonefritis proliferativas

\section{Lupus Nephritis}

\begin{abstract}
Lupus nephritis (LN) occurs between 30 and $70 \%$ of patients with systemic lupus erythematosus depending on race and sex; it occurs early in the disease and severe forms predominate, classes III, IV and mixed (V + III IV or V +). $50 \%$ of adults and $70 \%$ of Colombian children with lupus suffer NL sometime in life; in this population $25 \%$ of children and $38 \%$ of adults have nephrotic syndrome, remission rate at six months is low and nephrotic range proteinuria and elevated baseline creatinine predict failure to achieve remission at six months.
\end{abstract}

Keywords: Lupus, Nephritis, Nephrotic Syndrome, Proliferative glomerulonephritis

\section{Introducción}

L

a nefritis lúpica (NL) se define, según el ACR (American College of Rheumatology) ${ }^{1}$, sus guías de $\mathrm{NL}^{2}$ y las recomendaciones EULAR/ERA-EDTA (European League Against Rheumatism and European Renal Association-European Dyalisis and Trasplant Association) ${ }^{3}$, como la presencia de proteinuria persistente $>500 \mathrm{mg} / 24$ horas o $3+$ en muestra de orina ocasional o la presencia de cilindros celulares (hemáticos, granulosos, tubulares o mixtos). El grupo SLICC (Systemic Lupus Erythematosus International Collaboratiing Clinics) ${ }^{4}$ la define por la presencia de proteinuria $\geq 500 \mathrm{mg} / 24$ horas o proteinuria/creatinuria (UPCR) $\geq 50 \mathrm{mg} / \mathrm{mmol}$ o cilindros eritrocitarios y propone que la presencia de una biopsia renal compatible con NL más la presencia de anticuerpos antinucleares (ANA) o antiDNA, son criterio suficiente para clasificar un paciente como lúpico. 
La frecuencia de NL varía dependiendo de la raza, sexo y edad; en la cohorte EUROLUPUS ${ }^{5}$, con 1.000 pacientes, $97 \%$ de ellos caucásicos, $16 \%$ tenía NL al inicio de la enfermedad y $36 \%$ durante su evolución. En general la NL se presenta en el $30 \%$ de los pacientes lúpicos de raza blanca ${ }^{6}$ y el $60 \%$ de los afroamericanos ${ }^{7}$. En la cohorte GLADEL ${ }^{8}$ (Grupo Latinoamericano para el Estudio del Lupus) el $51.7 \%$ de los pacientes presentó NL, $58 \%$ de los mestizos y afro-latinoamericanos y $43.6 \%$ de los blancos latinoamericanos. Similares proporciones se describen en la cohorte LUMINA (Lupus in Minories Nature or Nurture $)^{9}$. En Colombia $50-55 \%$ de los adultos ${ }^{10-12}$ y $75 \%$ de los niños ${ }^{13}$ con lupus eritematoso sistémico (LES) sufren NL en algún momento de su evolución.

Entre 10 y $25 \%$ de los adultos con NL progresan a insuficiencia renal crónica terminal (IRCT) ${ }^{14,15}$. Las formas proliferativas son las más graves, comunes y las que más llevan a IRCT ${ }^{16}$.

En Colombia no hay estudios prospectivos que determinen el porcentaje de pacientes con NL que desarrollen IRCT, pero en varias cohortes predominan las formas histológicas graves ${ }^{10,11,17}$. Utilizando la clasificación histológica de la Organización Mundial de la Salud (OMS), en 2 cohortes de la ciudad de Medellín, encontramos predominio de las formas proliferativas. En una población de 46 pacientes con NL en el Hospital Universitario San Vicente de Paul ${ }^{11}$, el $46 \%$ tenía NL clase IV, $7.8 \%$ clase III, $9.3 \%$ formas mixtas (membranoproliferativas) y $15.6 \%$ clase $\mathrm{V}$ (membranosa pura). En un grupo de 32 pacientes con NL en la clínica León XIII ${ }^{10}$, la NL clase IV representó el $50 \%$, la III el $10 \%$ y la V el $19 \%$ de las biopsias.

Utilizando la clasificación de la Sociedad Internacional de Nefrología y de la Sociedad de Patología Renal, ISN/RPS, por sus siglas en inglés, en 140 biopsias del Hospital Pablo Tobón Uribe encontramos que el $83.87 \%$ de los casos tenía NL proliferativas (clase IV $63.75 \%$, III $13.42 \%$ y formas mixtas, $\mathrm{V} / \mathrm{III}$ o $\mathrm{V} / \mathrm{IV}, 6.7 \%)^{18}$; igualmente, en una población altamente seleccionada por gravedad y resistencia a la inmunosupresión estándar, tratada con rituximab (RTX), el 76\% de las pacientes tenían NL proliferativas $^{19}$.
El LES parece ser más grave en los niños y adolescentes; entre 60 y $80 \%$ presentan NL durante su evolución, las dos terceras partes con clases histológicas III o IV y entre 10 y 50\% llegan a IRCT ${ }^{20,21}$.

\section{Anatomía patológica}

La biopsia renal es de gran importancia en el enfoque diagnóstico de la $\mathrm{NL}^{16}$; la correlación clínico-patológica es inexacta ${ }^{11}$, por lo cual el resultado de la biopsia renal es útil para guiar el tratamiento, dar información sobre el pronóstico ${ }^{3,22,23}$ y descartar nefropatía por síndrome antifosfolípido $(\mathrm{SAF})^{24,25}$, microangiopatía trombótica (MAT) ${ }^{26}$ y glomerulopatía primaria.

Las indicaciones de biopsia renal varían de acuerdo a los grupos de trabajo; el ACR indica biopsia renal en pacientes con LES que presenten aumento de la creatinina sin causas alternativas como sepsis, hipovolemia o medicamentos, proteinuria confirmada $\geq 1$ gramo/24 horas o la combinación de proteinuria $\geq 500 \mathrm{mg} / 24$ horas y cilindros celulares o hematuria $\geq 5$ eritrocitos AP. Las guías EULAR/ERA-EDTA ${ }^{3}$ sugieren biopsia renal en todos los pacientes lúpicos con proteinuria reproducible $\geq 500 \mathrm{mg} / 24$ horas, especialmente si tienen hematuria glomerular o cilindros celulares.

El consenso del grupo de enfermedades autoinmunes sistémicas (GEAS) de la Sociedad Española de Nefrología (SEN) y la Sociedad Española de Medicina Interna (SEMI) ${ }^{23}$, indican la biopsia renal en todos los pacientes con LES que presenten deterioro inexplicado de la función renal, proteinuria confirmada $\geq 500 \mathrm{mg} / 24$ horas, UPCR $\geq 50 \mathrm{mg} / \mathrm{mmol}$ en muestra matutina o en orina de 24 horas o sedimento urinario activo. Los 3 grupos sugieren utilizar la clasificación ISN/RPS $2003^{27}$ (Tabla 1).

La clasificación ISN/RPS pretende unificar conceptos y homogeneizar los términos modificando la clasificación $\mathrm{OMS}^{28,29}$. Según esta nueva clasificación ${ }^{27}$, en la clase II pueden demostrarse depósitos subendoteliales o subepiteliales por microscopía electrónica o de inmunofluorescencia pero no de luz y las clases III y IV se subdividen en activa (A), crónica (C) y activa y crónica $(\mathrm{A} / \mathrm{C})$, dependiendo de las características de las lesiones histológicas; a su vez la 


\section{Tabla 1}

Clasificación histológica de la nefropatía lúpica según la Sociedad Internacional de Nefrología y

la Sociedad de Patología Renal 2003

\section{Clase I: Nefritis mesangial mínima}

Microscopía de luz normal con depósitos mesangiales en la inmunohistología y la microscopía electrónica

Clase II: Nefritis mesangial proliferativa

Ensanchamiento y/o proliferación mesangial en la microscopía de luz

Clase III: Nefritis proliferativa focal $(\mathbf{A}, \mathbf{A} / \mathbf{C}, \mathbf{C})$

Proliferación intracapilar en menos del 50\% de los glomérulos, con depósitos inmunes subendoteliales

Clase IV: Nefritis proliferativa difusa $(\mathrm{A}, \mathrm{A} / \mathrm{C}, \mathrm{C})$

Proliferación intracapilar en $50 \%$ o más de los glomérulos, con depósitos inmunes subendoteliales

Case V: Nefritis membranosa

Depósitos inmunes subepiteliales; pueden coexistir las clases II, III o IV

Clase VI: Nefritis esclerosante avanzada

Esclerosis global en más del $90 \%$ de los glomérulos

NL IV se subdivide en global ( $\geq 50 \%$ de los glomérulos comprometidos tienen lesiones globales) y segmentaria ( $\geq 50 \%$ de los glomérulos comprometidos tienen lesiones segmentarias). El grupo de trabajo ISN/RPS propone que todas las biopsias renales sean evaluadas por microscopía de luz (hematoxilina \& eosina, plata - metenamina, PAS y tricrómico), de inmunofluorescencia (IgG, IgA, IgM, C3, C1q, cadenas livianas kappa y lambda) y electrónica.

Todas las clasificaciones de NL enfatizan en los hallazgos glomerulares y vasculares por lo cual siempre deben complementarse con los índices de actividad (IA) y cronicidad (IC) que, además de incluir los hallazgos tubulointersticiales, de gran importancia para el pronóstico, dan información semicuantitativa de la anatomía patológica renal ${ }^{30}$.

Si bien la clasificación ISN/RPS es utilizada por la gran mayoría de los grupos, hay varios aspectos críticos a esta: los estudios de pronóstico fueron hechos con base en la clasificación OMS, la presencia de un solo glomérulo con lesiones activas o crónicas es suficiente para clasificar las lesiones proliferativas como $\mathrm{A} \mathrm{o} \mathrm{C}^{31}$, la diferenciación entre las formas globales y segmentarias no siempre es fácil y es de gran importancia en la fisiopatología y el pronóstico de la NL, y no incluye las lesiones tubulares ni el compromiso arteriolar.

En general, las clases histológicas I y II tienen un curso indolente y las III y IV (figuras 1 y 2), sin tratamiento, cursan con daño renal progresivo ${ }^{32}$, sin embargo, es frecuente la transformación histológica ${ }^{27}$; el $66 \%$ de los pacientes con NL III y $18 \%$ con NL $\mathrm{V}$ (figura 3) pasan a clase IV en forma espontánea o a formas menos graves con el tratamiento inmunosupresor. La probabilidad de duplicar la creatinina, llegar a IRCT o morir es mayor en las formas proliferativas ( $32 \%$ clase IV, $30 \%$ clase III, $18 \%$ clase V, $5 \%$ clase II, $\mathrm{p}<0.025)$ y en aquellos con índices de actividad (IA $7 \pm 6$ vs. $5 \pm 5, \mathrm{p}<0.05$ ) y cronicidad (IC $4 \pm 3$ vs. $2 \pm 2$ ) altos ${ }^{33}$. Los pacientes con proliferación extracapilar (medialunas) en fase celular y fibrosis intersticial son considerados de alto riesgo de IRCT (70\% a 90 meses, $\mathrm{p}<0.0001$ vs. los pacientes $\sin$ estos hallazgos $)^{34}$. Ningún estudio evalúa las diferencias en el pronóstico de los pacientes con NL II con o sin depósitos subepiteliales o subendoteliales.

Un aspecto muy importante para evaluar el pronóstico y definir el tratamiento de los pacientes con NL es la coexistencia de nefropatía por SAF. Las lesiones características de esta son la MAT (lesiones agudas) y la hiperplasia fibrosa de la íntima (lesiones vasculares crónicas), pero incluyen también aterosclerosis, trombosis organizada y atrofia cortical focal subscapular isquémica ${ }^{35,36}$; en una cohorte francesa el 32\% de los pacientes con NL tenía superpuestos, hallazgos histológicos sugestivos de nefropatía por $\mathrm{SAF}$, sin manifestaciones sistémicas de $\mathrm{SAF}$ en el $22 \%$ de los casos. Los hallazgos histológicos anotados se asociaron a SAF sistémico, anticoagulante lúpico, HTA, fibrosis intersticial y elevación de creatinina. En una cohorte colombiana de pacientes con $\mathrm{SAF}^{37}$ el $16 \%$ de los pacientes tenía algún tipo de nefropatía (NL 11\%, MAT 1\% y glomerulonefritis proliferativa difusa con patrón "full house" y serología para LES persistentemente negativa $1 \%)^{38}$.

LospacientesconLESpuedenpresentarMATenelcontexto de una púrpura trombótica trombocitopénica ${ }^{39}$ o tenerla solo a nivel renal, la cual puede estar 


\section{Figura 1}

Glomerulonefritis proliferativa difusa IV-G (inmunofluorescencia)

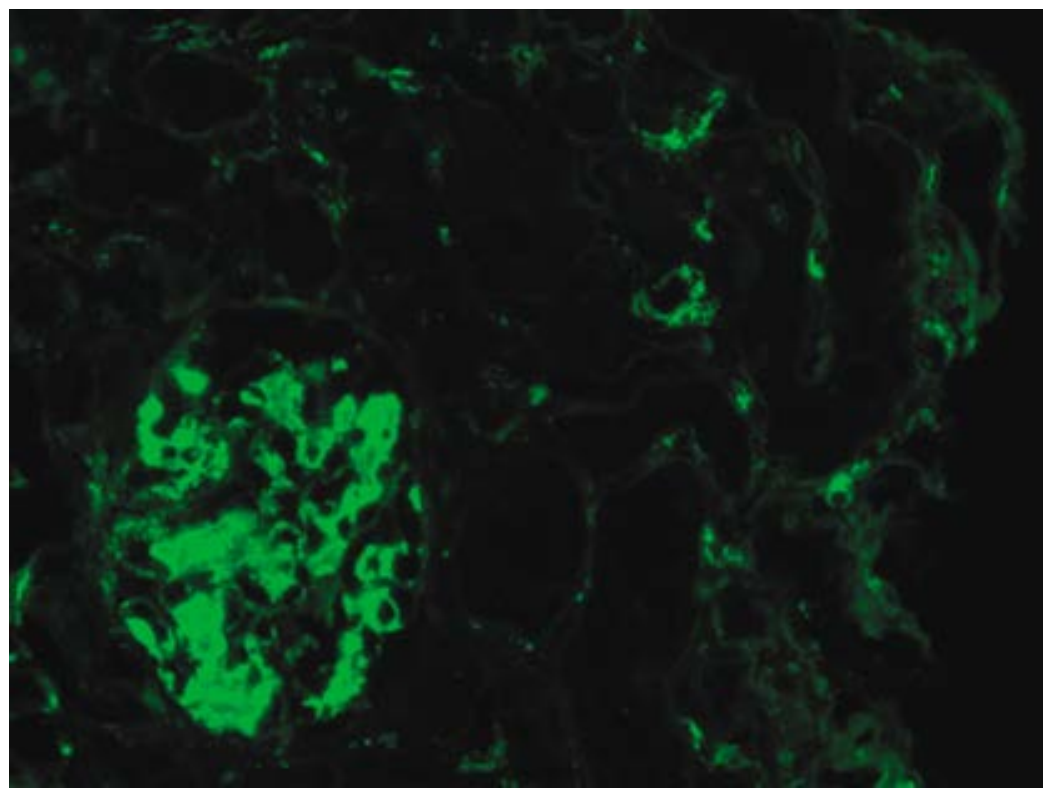

Figura 2

Nefritis lúpica proliferativa: depósitos inmunes subendoteliales (Microscopía electrónica)

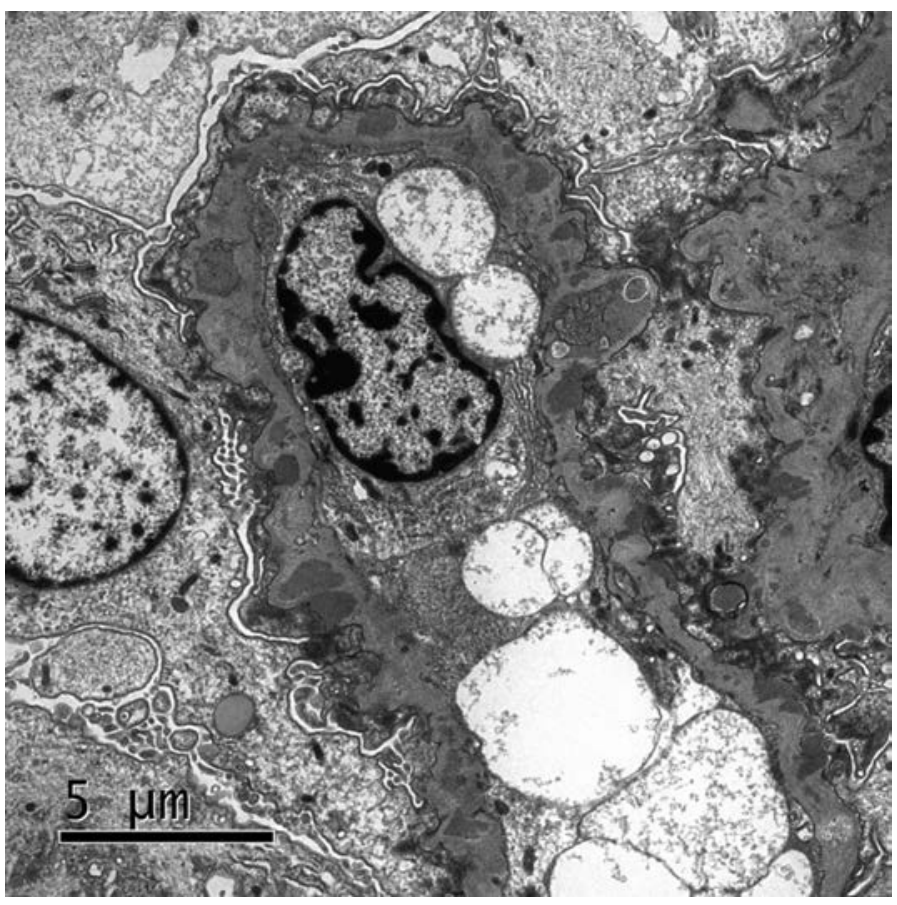




\section{Figura 3}

Nefritis lúpica V: Depósitos inmunes mesangiales, intramembranosos y subepiteliales y expansión del mesangio (Microscopía electrónica)

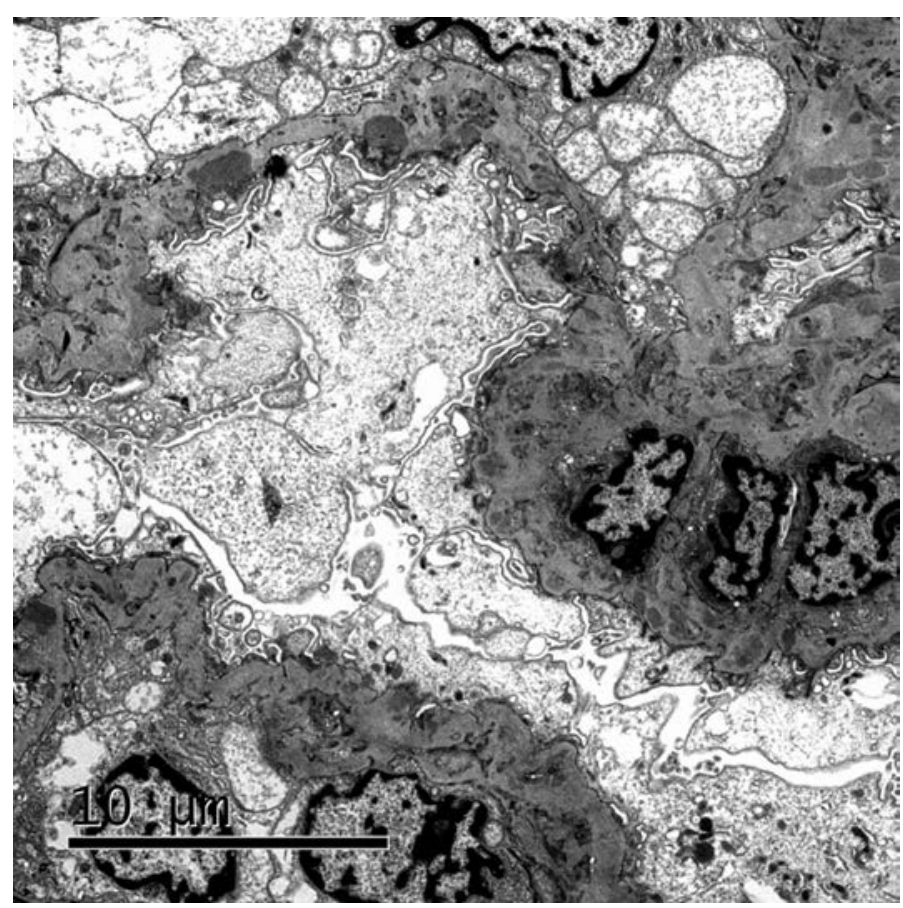

presente hasta en el $8.3 \%$ de las biopsias ${ }^{40}$. Los pacientes con MAT renal cursan con cuadros clínicos más graves ${ }^{41}$, con más proteinuria, mayor frecuencia de falla renal, HTA e hipertensión maligna y peor pronóstico a corto y largo plazos con mayor riesgo de IRCT y muerte. Solo el $60.6 \%$ cursan con anemia hemolítica microangiopática ${ }^{41}$, por lo cual se debe buscar la presencia de trombos en el glomérulo en todos los casos.

\section{Subgrupos especiales}

Tres aspectos adicionales de la histopatología deben ser analizados al revisar la biopsia renal de los pacientes con LES: la glomerulonefritis pauciinmune, focal y segmentaria, la glomerulonefritis crescéntica y las lesiones vasculares.

En la NL focal y segmentaria se afecta solo una porción de los glomérulos y del penacho glomerular, mientras que los otros no están afectados; con frecuencia los depósitos inmunes están ausentes de las lesiones focales semejando las pauciinmunes encontradas en las vasculitis asociadas a $\mathrm{ANCA}^{41-43}$. Algunos autores encuentran que los pacientes clasificados por OMS como clase III pero con afección segmentaria grave ( $\geq 50 \%$ de los glomérulos) tienen peor pronóstico que los pacientes con NL clase IV $^{44,45}$. Por definiciones de la clasificación ISN/RPS esos pacientes podrían quedar clasificados como IV-G siendo en realidad formas segmentarias extensas, con diferentes fisiopatología y pronóstico. Schwartz et al., ${ }^{43}$ evaluaron 83 biopsias renales con formas graves de NL que tuvieran proliferación o necrosis en $\geq 50 \%$ de los glomérulos no escleróticos y clasificadas por OMS como: segmentaria grave (clase III con afección $\geq 50 \%$ de los glomérulos), difusa global (clase IV) y membranosa (V \pm III y $\mathrm{V} \pm \mathrm{IV}$ ). Reclasificaron estas biopsias a la terminología ISN/RPS y encontraron que $26.5 \%$ de los casos tenía afección segmentaria que comprometía $>50 \%$ del penacho glomerular en $\geq 50 \%$ de los glomérulos. Aunque tenían afección de más del $50 \%$ del penacho glomerular, en realidad estos pacientes tenían formas 
segmentarias extensas, no globales. La importancia de esto radica en que este subgrupo de pacientes tuvo menor tasa de remisión, supervivencia renal, estabilidad de la función renal y mayores tasas de IRCT y mortalidad que la glomerulonefritis IV-G y IV-S. Hay diferencias histológicas que podrían explicar estos hallazgos; las biopsias con glomerulonefritis segmentaria grave tienen menos depósitos inmunes subendoteliales, trombos hialinos y asas de alambre que la glomerulonefritis proliferativa global, es decir, son más pauciinmunes, sin embargo, tienen más daño de la pared capilar ${ }^{44}$.

Los pacientes con glomerulonefritis rápidamente progresiva (GMNRP) representan un subgrupo aparte; típicamente tienen falla renal acelerada con pérdida de la función renal en $\leq 3$ meses, evidencia de injuria glomerular con sedimento urinario activo y proliferación extracapilar ${ }^{46}$. La caracterización de estas biopsias por inmunohistología muestra 3 patrones: Tipo 1: patrón granular, característico de las glomerulopatías por complejos inmunes, Tipo 2: patrón lineal, antimembrana basal glomerular y Tipo 3: pauciinmune, observado en las vasculitis asociadas a ANCA y la glomerulonefritis crescéntica pauciinmune.

Siendo LES una enfermedad con alta actividad de la inmunidad humoral se esperaría que los pacientes con GMNRP tuvieran el Tipo 1, sin embargo se describen formas graves de GMNRP con prominentes necrosis fibrinoide y proliferación extracapilar con depósitos inmunes subendoteliales mínimos o ausentes y ANCA positivos ${ }^{47,48}$. La glomerulonefritis crescéntica no es rara en el lupus, se observa en el $10 \%$ de todas las biopsias renales y en el $21.7 \%$ de las NL IV-G ${ }^{49}$; los ANCA podrían tener un papel patogénico en las nefritis crescéntica y necrosantes segmentarias ${ }^{45-50}$. Los pacientes con formas crescénticas tienen menor probabilidad de remisión parcial o completa y mayor riesgo de recaer, duplicar la creatinina, tener ANCA positivos y sufrir IRCT; además cursan con más inflamación y fibrosis intersticial, atrofia tubular e índices de actividad y cronicidad ${ }^{47}$.

Otro aspecto importante para el pronóstico de la NL es el compromiso vascular no glomerular ${ }^{50}$. Appel y et al., ${ }^{51}$ describieron, por primera vez, el término vasculopatía lúpica como una microangiopatía necrosante renal no inflamatoria, sin trombos ni asociación a SAF (figura 4). En un estudio de 169 biopsias renales de pacientes franceses con $\mathrm{NL}^{52} \mathrm{se}$ encontró arterioloesclerosis (49.7\%), depósitos inmunes en la pared vascular (30.2\%), vasculopatía lúpica $(24.3 \%)$, vasculitis $(2.4 \%)$ y MAT $(0.6 \%)$. Los pacientes con compromiso vascular tenían mayor porcentaje de NL IV OMS, más HTA, anemia, falla renal, proteinuria, síndrome nefrótico, hipocomplementemia, anemia y trombocitopenia que aquellos sin afección vascular. En pacientes chinos ${ }^{43,53,54}$ la distribución de las formas de afección vascular fue diferente: $66 \%$ tenían depósitos inmunes vasculares, $12.6 \%$ arterioloesclerosis, $12 \%$ MAT, $6.3 \%$ vasculopatía necrosante no inflamatoria, $1.4 \%$ vasculitis y $28 \%$ más de 2 formas de lesión vascular. Los pacientes con vasculopatía lúpica presentaron cuadros histológicos más graves, mayores IA e IC, hipercelularidad endocapilar, cariorrexis, necrosis fibrinoide, depósitos subendoteliales, atrofia tubular y fibrosis intersticial; además cursaron con más citopenias hematológicas, hipocomplementemia y retención de azoados.

En los pacientes con proteinuria de baja magnitud, al igual que en el resto de los casos, la correlación clínico - patológica es inexacta y podría tratarse de NL grave en fases iniciales. En la cohorte de John Hopkins $^{55}$ evaluaron los hallazgos de 21 biopsias renales hechas mientras los pacientes tenían proteinuria $<1$ gramo/24 horas o UPCR $<1$. En este grupo, que representó el $6.4 \%$ de la muestra, encontraron 5 casos de NL clase III, una clase IV, una clase V y 6 clase $\mathrm{V}$ asociadas a III o IV; además una biopsia mostró MAT. La única diferencia entre los pacientes con formas histológicas graves y leves fue la hipocomplementemia en las primeras. Entonces, pacientes con LES con protenuria leve, sedimento limpio y función renal normal pueden tener formas histológicas graves. La hipocomplementemia C3 podría sugerir la presencia de glomerulonefritis proliferativas en este subgrupo ${ }^{56}$.

\section{Manifestaciones clínicas y factores predictores de remisión}

La NL se manifiesta con 6 diferentes patrones clínico-patológicos: proteinuria dominante con o sin 


\section{Figura 4}

Vasculopatía lúpica: depósito masivo de complejos inmunes y C4 en los capilares peritubulares

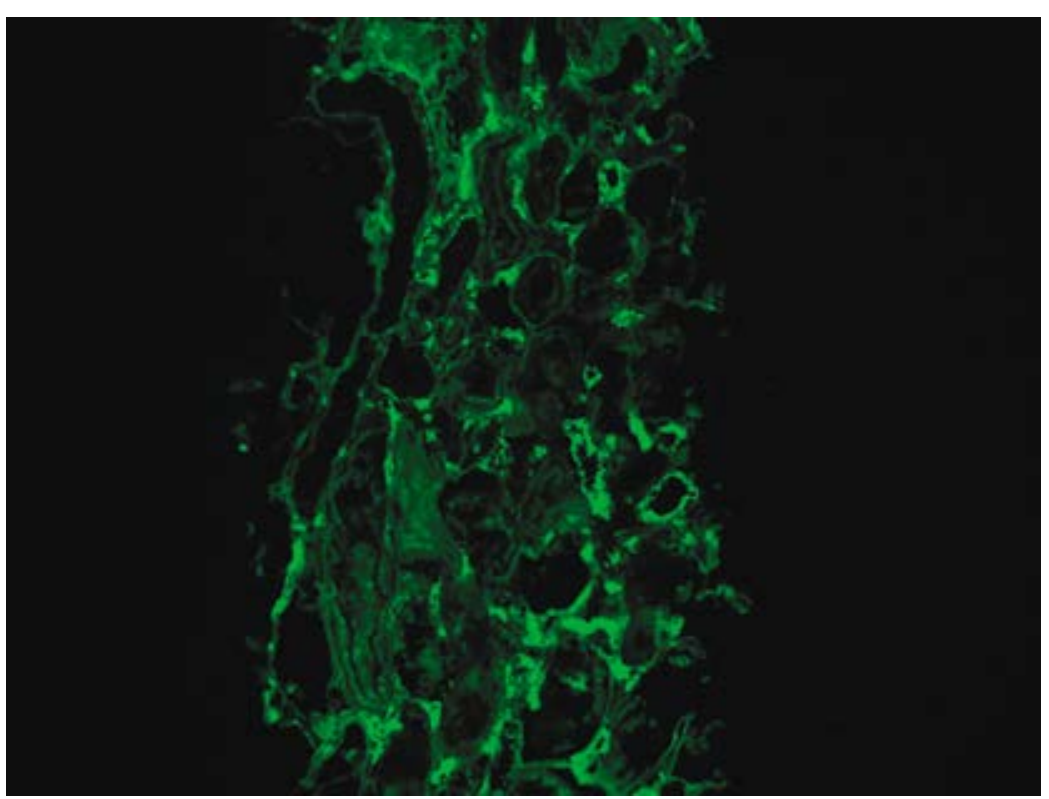

sedimento telescopado, insuficiencia renal aguda o crónica, síndrome nefrítico con o sin falla renal, GMNRP, síndrome nefrótico y MAT agregada ${ }^{16}$. En nuestro medio la NL se presenta temprano en la historia natural de la enfermedad. En una cohorte de 56 niños colombianos $16.1 \%$ tenía síndrome nefrítico y $14.3 \%$ síndrome nefrótico al diagnóstico del LES. En una población de 104 adultos colombianos con $\mathrm{LES}^{10}, 35.7 \%$ tenía nefritis al diagnóstico. En una cohorte de 84 pacientes de la ciudad de Mede1 lín ${ }^{17}$ observamos que la NL se presentó en promedio a los $13.6(0-168)$ meses después del diagnóstico de LES. El diagnóstico de LES y NL coincidieron en el $36.9 \%$ de los pacientes, el $63 \%$ de los que hicieron NL la presentaron dentro de los 6 primeros meses y el $72 \%$ durante el primer año.

La frecuencia de síndrome nefrótico varía en los diferentes grupos étnicos; en las series colombianas el $25 \%$ de los niños ${ }^{13}$ y hasta el $38 \%$ de los adultos ${ }^{10,11,17}$ cursan con este. En la cohorte GLADEL $^{8}, 5.7 \%$ de los blancos, $6.7 \%$ de los mestizos y $10.5 \%$ de los afrolatinoamericanos con LES presentaron síndrome nefrótico. Esta manifestación es más frecuente en los pacientes lúpicos afroamericanos ${ }^{6}$ y menos frecuente en los caucásicos ${ }^{57}$. En nuestro medio el $40 \%$ de los pacientes con NL III, $41 \%$ de NL IV, $50 \%$ de las formas mixtas y $70 \%$ de la NL V pura, cursaron con proteinuria en rango nefrótico ${ }^{11}$.

Un aspecto muy importante en el enfoque inicial de los pacientes con NL es detectar los factores predictores de respuesta al tratamiento, duplicación de la creatinina, IRCT y muerte. Los niveles basales de creatinina y proteinuria, HTA, anticuerpos anti-DNA, hipocomplementemia $\mathrm{C} 3$ y $\mathrm{C} 4$, altos IA e IC, raza hispana y afroamericana son factores de mal pronóstico en pacientes con NL proliferativa ${ }^{16,32,58-65}$. La falla en el logro de remisión parcial (RP) o completa (RC) a los 6 meses se asocia a mal pronóstico a largo plazo e implica mayor uso de inmunosupresores ${ }^{16}$. En pacientes asiáticos el tiempo más largo en lograr remisión y el no lograr RC son predictores de recaída e $\mathrm{IRCT}^{66}$. Por otra parte la normalización de la creatinina en las primeras 48 semanas $^{67}$, de la proteinuria en las primeras $52^{68}$ y la disminución de esta a menos de 1 gramo/24 horas en las primeras $24^{69}$, fueron predictores de buen pronóstico en pacientes caucásicos con NL. 
En una cohorte pacientes colombianos con NL proliferativas tratados con esteroides y ciclofosfamida (CFM) o micofenolato mofetil (MMF) ${ }^{17}$ solo el 44\% alcanzaron RP o RC a los 6 meses $(23.8 \% \mathrm{RC}$ y $20.2 \% \mathrm{RP}$ ); el $52.7 \%$ de las mujeres y $80 \%$ de los hombres fallaron en el logro de RP o RC a los 6 meses; el $23.4 \%$ de los que fallaron, alcanzan al menos $\mathrm{RP}$ a los 12 meses. En el análisis multivariado la alteración basal de la función renal (OR 10.92, 95\% IC $2.65-45.02, \mathrm{p}=0.001)$ y la proteinuria en rango nefrótico (OR 9.81, 95\% IC 1.85 - 52-04, $\mathrm{p}=0.007$ ) fueron predictores independientes de falla en el logro de RP o RC a 6 meses.

\section{Tratamiento}

Los objetivos del tratamiento de la NL son RC, o al menos RP, a los 6 meses y no después de 12 meses, evitar el daño renal irreversible, diálisis, trasplante y muerte, siempre buscando la menor toxicidad posible por medicamentos y preservando la calidad de vida $^{3}$.

Los estudios que evalúan los desenlaces RC y RP son muy heterogéneos por diferentes definiciones de respuesta. El ACR define RC como tasa de filtración glomerular (TFG) $>90 \mathrm{~mL} / \mathrm{min} / 1.73 \mathrm{~m} 2 \mathrm{de}$ superficie corporal, proteinuria $<500 \mathrm{mg} / 24$ horas (o UPCR $<50 \mathrm{mg} / \mathrm{mmol}$ ) y sedimento urinario limpio $(<5 \text { eritrocitos o leucocitos AP })^{70}$. Las guías EULAR/ERA-EDTA proponen criterios similares de $\mathrm{RC}$ y RP pero definen respuesta de la función renal como normal o "casi normal" (TFG máximo $10 \%$ por debajo de lo normal) $)^{3}$.

Los esquemas de inmunosupresión actuales constan de una fase de inducción y una de mantenimiento ${ }^{2,3}$. El tratamiento de inducción se hace con esteroides en combinación con inmunosupresores, MMF o CFM. El esquema sugerido de esteroides es metilprednisolona endovenosa $500 \mathrm{mg} /$ día por 3 dosis (1 gramo si se demuestra proliferación extracapilar) seguido de prednisolona $0.5-1 \mathrm{mg} / \mathrm{kg} /$ día en dosis decrecientes. Si se elige MMF la dosis recomendada es de $2-3$ gramos/ día. Si se elige CFM hay 2 formas de administración: $0.75-1$ gramo $/ \mathrm{m}^{2} / \mathrm{mes}$ durante 6 meses (esquema del Instituto Nacional de Salud de Estados Unidos, NIH) ${ }^{71}$ o $750 \mathrm{mg}$, dosis fija, cada 15 días por 6 dosis ("esquema EUROLUPUS") 57 .
Las guías EULAR/ERA-EDTA ${ }^{3}$ solo recomiendan el segundo esquema.

Los estudios clásicos del $\mathrm{NIH}^{72,73}$ nos dejaron varias enseñanzas: en el tratamiento de la NL los esteroides solos son inferiores a su combinación con citotóxicos, azatioprina (AZA) es inferior a CFM en la inducción de remisión, los esquemas cortos (6 meses) con CFM son inferiores a largo plazo a los esquemas largos (24 meses), la CFM en pulsos mensuales o trimestrales confiere menor toxicidad que la administración oral diaria y los pulsos de metilprednisolona ayudan a un uso posterior de menores dosis de esteroides. El uso de CFM por períodos de 2 años se asoció a mayor riesgo de falla ovárica prematura, leucopenia, infecciones y displasia del cuello uteri$\mathrm{no}^{72}$. Como una alternativa con menor toxicidad, el estudio EUROLUPUS mostró resultados similares aunque el comparador no fue el esquema NIH e incluyó solo pacientes caucásicos ${ }^{57,69,74}$.

Los estudios clínicos controlados (RCT) que compran MMF y CFM en inducción de remisión de NL proliferativa ${ }^{71,74-78}$ han sido evaluados en 2 recientes metaanálisis ${ }^{79,80}$ que no demostraron diferencias en el logro de RP, RC y estabilización de la función renal; CFM causa más alopecia y falla ovárica y MMF más diarrea, pero no hubo diferencias en la presentación de herpes zoster e infecciones mayores y el riesgo de IRCT y muerte fue igual para ambos medicamentos.

En la elección entre CFM y MMF influyen factores como la edad ${ }^{21}$, raza $^{75}$, futuro reproductivo y gravedad del cuadro clínico. Si bien la mayoría de los clínicos prefieren CFM para casos graves, recientemente se publican resultados exitosos de tratamiento de NL proliferativas con alteración de la función renal, vasculopatía y glomerulonefritis crescéntica, con $\mathrm{MMF}^{81-83}$. Micofenolato sódico ${ }^{80}$ y tacrólimus ${ }^{84}$ también han sido evaluados en inducción de remisión de NL proliferativa con resultados favorables.

Según las recomendaciones EULAR y ACR los pacientes que no hayan logrado RP o RC a los 6 meses deben ser sometidos a reinducción con metilprednisolona seguida de dosis plenas de prednisolona combinada con CFM o MMF (el que no fue elegido en el tratamiento inicial). Cuando hay falla a la reinducción se sugiere tratamiento con RTX o ciclosporina $\mathrm{A}(\mathrm{CsA})$. 
Los pacientes que logran RP o RC pasan a fase de mantenimiento con MMF (1 - 2 gr/ día) o AZA (2 $\mathrm{mg} / \mathrm{kg} / \mathrm{día})$; la terapia de mantenimiento se debe continuar al menos por 3 años para evitar recaídas $^{2,3,85}$. Los estudios que comparan MMF y AZA en mantenimiento ${ }^{86-89}$, agrupados en un reciente metaanálisis ${ }^{90}$, no mostraron diferencias en las tasas de recaída, IRCT y muerte. Los tratados con AZA presentaron más leucopenia y amenorrea pero no hubo diferencia en los eventos adversos gastrointestinales e infecciones. La extensión del estudio ASPREVA ${ }^{87}$ favorece a MMF y el estudio MAINTAIN ${ }^{88}$ a AZA.

Rituximab en la inducción de remisión de NL proliferativa ha sido evaluado solo en un estudio controlado aleatorizado (RCT por sus siglas en inglés) ${ }^{91}$ que no demostró que fuera mejor que el placebo en el logro de respuesta parcial o completa en pacientes que recibían esteroides y MMF. Los pacientes del grupo RTX tuvieron más disminución $\geq 50 \%$ de la proteinuria $(\mathrm{p}=0.04)$, necesitaron menos rescates con CFM ( $p=0.006)$ y lograron mayor ahorro de esteroides, negativización de los anticuerpos anti-DNA y normalización de los niveles de C3.

Los estudios "de la vida real"92-100, en su mayoría de cohortes, muestran resultados diferentes al estudio LUNAR; la mayoría de los pacientes incluidos recibieron RTX por falla al manejo inmunosupresor, algunos en combinación con MMF o CFM y otros como monoterapia. La principal conclusión de estos estudios es que RTX estaría indicado en casos refractarios de NL proliferativa. Esta es la indicación que acogen las recomendaciones ACR y EULAR/ ERA-EDTA. En pacientes colombianos ${ }^{19}, 75 \%$ de ellos con NL refractaria a 2 o más inmunosupresores, se observó mejoría significativa de la proteinuria, depuración de creatinina, actividad lúpica medida por SELENA-SLEDAI y disminución de los requerimientos de esteroides. A los 12 meses del tratamiento con RTX $61.5 \%$ de los pacientes lograron $\mathrm{RP}$ o $\mathrm{RC}$ por el parámetro proteinuria y el $33 \%$ por el parámetro depuración de creatinina. El 20\% de los pacientes requirió retratamiento con RTX, en promedio a los 44 meses (95\% IC 10.1 - 50.1).

Para pacientes con NL refractaria y MAT asociada, se han utilizado recambios plasmáticos y en casos de NL activa e infección agregada, gammaglobulina endovenosa. Ninguno de estos tratamientos ha sido evaluado en RCT, solo en estudios abiertos ${ }^{80}$.

La glomerulonefritis membranosa con clase III o IV asociadas, se tratan como NL proliferativa; las formas $\mathrm{V}$ puras representan entre 0 y $19 \%$ de los casos publicados en Colombia ${ }^{10-13}$. Las guías ACR2 y EULAR/ERA-EDTA ${ }^{3}$ recomiendan iniciar con prednisolona $0.5 \mathrm{mg} / \mathrm{kg} /$ día y MMF o AZA. Si no hay respuesta favorable a los 6 meses sugieren pulsos de CFM y metilprednisolona. Para casos refractarios hay evidencia de efecto favorable con $\mathrm{CsA}^{101}$ y $\operatorname{RTX}^{93,94}$.

Las terapias coadyuvantes son muy importantes en el pronóstico de la NL; todos los pacientes con proteinuria $\geq 500 \mathrm{mg} / 24$ horas deben recibir inhibidores de la enzima convertidora de la angiotensina o bloqueadores de los receptores de angiotensina. Estos medicamentos logran disminuir hasta en un 30\% la proteinuria y retardar la duplicación de creatinina e IRCT en pacientes no diabéticos ${ }^{2,3}$. Se indican metas de presión arterial $\leq 130 / 80$ y control estricto de la dislipidemia. La hidroxicloroquina disminuye el daño acumulado incluido el renal y las recaídas, por lo cual debe ser recibida por todos los pacientes con LES y NL excepto si hay contraindicaciones ${ }^{102,103}$.

\section{Colaboradores:}

Adriana Flórez Vargas, nefropatóloga, Fundación Santa Fe de Bogotá y Mauricio Lopera, nefrólogo RTS - Baxter Medellín, por las imágenes histopatológicas.

\section{Agradecimientos}

A los doctores Carolina Muñoz Grajales, Javier Darío Márquez Hernández y Carlos Jaime Velásquez Franco, reumatólogos del Hospital Pablo Tobón Uribe por su valiosa participación en las investigaciones en nefritis lúpica en la ciudad de Medellín.

\section{Conflictos de interés y fuentes de financiación:}

El autor no recibió financiación para la realización de esta revisión y no tiene conflictos de interés que declarar. 


\section{Bibliografía}

1. Tan EM, Cohen AS, Fries JF, Masi A, Mc Shane D, Rothfield N, et al. The 1982 revised criteria for classification Systemic Lupus Erythematosus. Arthritis \& Rheum 1982;25:1271-1277.

2. Hahn BH, Mc Mahon M, Wilkinson A, Wallace D, Daikh D, Fitzgerald J, et al. American College of Rheumatology guidelines for screening, treatment and manegement of Lupus Nephritis. Athritis Care Res. 2012;64:797-808.

3. Bertsias G, Tektonidou M, Amoura Z, Aringer M, Bajema I, Benden J, et al. Joint European Leage Against Rheumatism and European Association- European Dyalisis and Trasplant Association (EULAR/ERA-EDTA) recommedations for the manegement of adult and pediatric Lupus Nephritis. Ann Rheum Dis 2012;71:1771-1782.

4. Petri M, Orbai AM, Alarcón GS, Gordon C, Merrill JT, et al. Derivation and Validation of the Systemic Lupus Erythematosus international collaborating clinics classification criteria for Systemic Lupus Erythematosus. Arthritis Rheum 2012;64:2677-2686.

5. Cervera R, Khamastha M, Font J, Sebastiani JD, Gil A, La Villa P, et al. Systemic Lupus Erythematosus: Clinical and Immunologic patters of disease expression in a cohort of 1000 patients. Medicine 1993;72:113-124.

6. Arbuckle MR, James JA, Denis GJ, Rubertone MV, Mc Clain MT, et al. Rapid clinical progression to diagnosis among African-American men with Systemic Lupus Erythematosus. Lupus 2003;12:99-106.

7. López P, Mozo L, Gutiérrez C, Suárez A. Epidemiology of Systemic Lupus Erythematosus in a northern Spanish Population: Gender and Age influences in immunologic features. Lupus 2003;12:860-865.

8. Pons-Estel B, Catoggio L, Cardiel M, Soriano E, Gentilitti S, Villa A, et al. The GLADEL multinational Latin American Prospective Inception Cohort of 1214 patients with Systemic Lupus Erythematosus. Medicie 2004;83:1-18.

9. Bertoli A, Alarcón GS, Calvo-Alen J, Fernández M, Vila L, Reveille J, for the LUMINA study group. Systemic Lupus Erythematosus in a multicentric US cohort. Clinical features, course and outcome kin patients with late onset disease. Arthritis Rheum 2006;54:1560-1567.

10. Pinto LF, Guerra L, González JR, Pérez A, Velásquez CJ, Felipe O, et al. Lupus Eritematoso Sistémico: análisis del comportamiento clínico en una población de Medellín. Rev Col Reum 1997;4:170-173.

11. Senior JM, Pinto LF, Uribe O, Felipe O, Ramírez LA. Nefritis Lúpica: Correlación clínico-patológica y respuesta a pulsos de ciclofosfamida. Rev Col Reum 1994;1:19-27.

12. Ruiz O, Londoño J, Vélez P, Ortiz I, Motta L, Valle R. Descripción de una cohorte de pacientes con Lupus Eritematoso Sistémico (LES) en un hospital de Bogotá-Colombia. Rev Col FReum 2003;10:266-275.

13. Ramírez-Gómez LA, Builes CA, Maya C, Palacio CA, Velásquez JF, Uribe O. Lupus Eritematoso Sistémico en niños. Rev Col Reum 1998;5:11-17.

14. Martins L, Rocha G, Rodríguez A, Santos J, Vasconcelos C, Correia J, et al. Lupus Nephritis: A restrospective review of 78 cases from a single center. Clin Nephrol 2002;57:114-119.

15. Mc Gowan JR, Ellis S, Griffiths M, Isenberg D. Retrospective analysis of outcome in a cohort of patients with Lupus Nephritis treated betwen 1997 and 1999. Rheumatology 2002;41:981-987.

16. Ortega LM, Schultz DR, Lens O, Pardo V, Contreras G, et al. Lupus Nephritis: Pathologic features, epidemiology and guide to therapeutic decisions. Lupus 2010;19:567-574.

17. Pinto LF, Castro IL, Duque V, Márquez J, Velásquez CJ. Factores de riesgo predictores de falla a la terapia de inducción de nefritis lúpica en una cohorte de pacientes colombianos. Reumatología Clínica 2014;10:147-151.

18. Pinto LF, Márquez JD, Velásquez CJ, Duque V. Lupus Nephritis: Description of a cohort of hispanic patients and detection of remission predictors at 12 months. Lupus 2013;22:1-86,P094.

19. Pinto LF, Velásquez CJ, Prieto C, Mestra L, Forero E, Márquez JD. Rituximab induces a rapid and sustained remission in colombian patients with severe and refractory Systemic Lupus Erythematosus. Lupus 2011;20:1219-1226.

20. Sunder R, Solomons, Lisk L, for the Aspreva Lupus Management Study (ALMS) group. Efficacy of Mycophenolate Mofetil in adolescent patients with Lupus Nephritis: Evidence from a two-fhase prospective randomized trial. Lupus 2012;21:1433-1443. 
21. Perfumo F, Martin A. Lupus Nephritis in children. Lupus 2005;14:83-88.

22. Markowitz GS, D agati VD. The ISN/RPS classification of the glomerulonephritis in Systemic Lupus Erythematosus Revisited. J Am Soc Nephrol 2004;15:241-250.

23. Ruiz-Irastorza G, Espinosa G, Frutos M, Jiménez-Alonso J, Praga M, Pallares L, et al. Diagnóstico y tratamiento de la nefritis lúpica. Nefrología 2012;32(SUPPL 1):1-35.

24. Nochi D, Daugas E, Droz D, Beausfils H, Grünfeld JP, Piette JC, et al. The intrarrenal vascular lesions associated with primary antiphospholid síndrome. Journal Of Am Soc Nephrol 1999;10:507-518.

25. Daugas E, Nochi D, Hupong D, Duchat P, Beausfil H, Cadwell V, et al. Antiphospholipid syndrome nephropaty in Systemic Lupus Erythematosus. Journal Of Am Soc Nephrol 2002;13:42-52.

26. Nesher G, Hanna VE, Moore TL. Thrombotic Microangiophatic Hemolitic Anemia in Systemic Lupus Erythematosus. Sem SArth Rheum 1994;24:165-172.

27. Weening JJ, Dàgati VD, Schwartz M, Seshan S, Alpers Ch, Appel G. The calassification of glomerulonephritis in Systemic Lupus Erythematosus. Kidney Int 2004;65:521-530.

28. Churg J, Sobin LH: Renal Disease. Classification and Atlas of glomerular diseases Tokio, Igaku - Shoin, 1982.

29. Churg J, Bernstein J, Glassock PJ. Renal Disease. Classification and Atlas of glomerular diseases 2nd Ed, New York,Igaku - Shoin 1995.

30. Pirani CL, Pollack VE, Schwartz FD: The Reproductibility of semicuantitative analysis of renal histology. Nephron 1964;1:230-237.

31. Haas M, Rastaldi M, Fernenz A. Histologic Classification of the glomerular diseases: clinic-pathologic correlation limitations exposed by validation studies and suggestions of modification. Kidney Int 2014;85:779-793.

32. Contreras G, Pardo V, Cely C, Borja E, Hurtado A, De la Cuesta C. Factors associated with poor outcomes in patients with Lupus Nephritis. Lupus 2005;14:890-895.

33. Najafi CC, Korbet M, S, Lewis E, Schwartz M, Reichlin M. Significance of histologic patterns of glomerular injury upon long-term prognosis in severe lupus glomerulonephritis. Kidney Int 2001;59:2156-2163.

34. Austin HA III, Boumpas DT, Vaughan EM, Ballow JE. High-risk features in Lupus Nephritis: importance of race and clinical and histological factors in 166 patients. Nephrol Dyal Trasplant 1995;10:1620-1628.

35. Taraborelli M, Andreoli L, Tincani A. Munch more tan thromboses and pregnancy loss: The Antiphospholipid Syndrome as a sistemic disease. Best Pract Clin Rheumatol 2012;26:79-90.

36. Alchi B, Griffiths M, Jayne D. Wath Nephrologist need to know about antiphospholipid syndrome. Nephrol Dyal Trasplant 2010;25:3147-3154.

37. Mesa M, Saldarriaga C, Aguilar C, Builes C, Quiroga A, Aristizabal N, et al. Síndrome antifosfolípido: descripción de una cohorte de pacientes colombianos y evaluación de los factores de riesgo para trombosis. Rev Col Reum 2012;19:208-217.

38. Granda P, Restrepo M, Velásquez CJ, Pinto LF, Márquez J, Mejía R et al. Hemorragia alveolar difusa y nefropatía en dos pacientes con síndrome antifosfolípido primario. Rev Col Reum 2011;16:68-74.

39. Espinosa G, Bucciarelli S, Cervera R, Lozano M, Reverter JC, de la Red G, et al. Thrombotic microangiophatic haemolytic anemia and antiphospholipid antibodies. Ann Rheum Dis 2004;63:730-736.

40. Hu WX, Liu ZZ, Chen HP, Zhang HT, Li S, Liou ZH. Clinical Characteristics and prognosis of diffuse proliferative Lupus Nephritis with thrombotic microangiopathy. Lupus 2010;19:1591-1598.

41. Bridoux X, Vrtovsnik F, Noel C, Saunier P, Mougenot B, Lemaitre V, et al. Renal Thrombotic microangiopathy in systemic lupus erythematosus. Clinical correlations and long-term renal survival. Nephrol Dyal Trasplant 1998;13:298:304.

42. Yao G, Hu WX, Liu ZH, Chen HP, Zhang HT, Li LS. The significance of renal vasculopathy in patients with diffuse proliferative lupus nephritis. Nephrol Dyal Trasplant 2002;11:211-219.

43. Schwartz M, Korbet S, Lewis E (For the collaborative study group) The prognosis and pathogenesis of severe Lupus Glomerulonephritis. Nephrol Dyal Tasplant 2008;23:1298-1306. 
44. Behara V, Whittier W, Korbet S, Schwartz M, Martens M, Lewis E. Pathogenetic features of severe segmental Lupus Nephritis. Nephro Dyal Trasplant 2010;25:153-159.

45. Gill GF, Dellahouse M, Nochy D, Bariety J. Class IV-S versus IV-G Lupus Nephritis: Clinical and morphologic differences suggesting different pathogenesis. Kidney Int 2005;68:2288-2297.

46. Masani N, Imbriano L, D’agati V, Markowitz G. SLE and Rapidly Progressive Glomerulonephritis. Am J Kid Dis. 2005;45:950-955.

47. Nasr S, D`agati V, Park HR, Sterman P, Goyzueta J, Dressler R, et al. Necrotizing and Crescentic Lupus Nephritis with Antineutrophil Cytoplasmic Antibody Seropositivity. Clin J Am Soc Nephrol 2008;3:682-690.

48. Markowitz GS, D`agati VA. Classification of Lupus Nephritis. Curr Op Nephrol Hypert 2009;18:220-225.

49. Yu F, Tan Y, Lui G, Wang SX, Zou WZ, Zhao MH. Clinicophatological characteristics and outcome of patients with crescentic lupus nephritis. Kidney Int 2009;76:307-317.

50. Abedallatif AA, Warris S, Lakhani A, Kadikoy H, Haque W, Truong LD. True vasculitis in Lupus Nephritis. Clin Nephrol 2010;74:106-112.

51. Appel GB, Pirani CL, D`agati V. Renal vascular complications in Systemic Lupus Erythematosus. J Am Soc Nephrol 1994;4:1499-1515.

52. Descombes E, Droz D, Drouet L, Grunfeld JP, Lesavre P. Renal vascular lesions in Lupus Nephritis. Medicine 1997;76:355-368.

53. Chu H, Wu LH, Song D, Yu F, Zhao MH. Non inflammatory necrotizing vasculopathy in Lupus Nephritis. A single center experience. Lupus 2014;23:20-30.

54. Wang J, Hu W, Xie H, Zhang H, Chen H, Zeng C, et al. Induction therapies for class IV Lupus Nephritis with non inflammatory necrotizing vasculopathy: Mycophenolate Mofetil or intravenous cyclophosphamide. Lupus 2007;16:707-712.

55. Christopher-Stine L, Siedner M, Lin J, Haas M, Perekh H, Petri M, et al. Renal biopsy in patients with low levels of proteinuria. J Rheumatol 2007;34:1491-1496.

56. Oates J. Renal biopsy at the onset of clinical lupus nephritis: Can it yield useful information? J Rheumatol 2007:34:256-257.

57. Houssiau F, Vaconcelos C, D`Cruz D, Sebastiani JD, De Ramón E, Danielli M, et al. Immunosuppresive therapy in lupus nephritis. The Eurolupus Nephritis Trial, a Randomized Trial of low-doses versus high-doses intravenous cyclophosphamide. Arthritis Rheum 2002;46:2121-2131.

58. Austin III HA, Boumpas D, Vaughan H, Ballow J. Predicting renal outcomes in severe lupus nephritis: contribution of clinical and histologic data. Kid Int 1994;45:544-550.

59. Arce-Salinas CA, Villa AR, Martínez-Rueda JO, Muñoz L, Cardiel MH, Alcocer-Varela J, et al. Factors associated with chronic Renal Failure in 121 patients with lupus nephritis a case-control study. Lupus 1995;4:197-203.

60. Korbet S, Lewis E, Schwartz M, Reichlin M, Evans J, Rhonde R, et al. Factors Predictive of outcome in severe lupus nephritis. Am J Kid Dis 2000;35:904-914.

61. Mok CC, Ho CT, Chan KW, Law GS, Wong RW. Outcome and prognostic indicators of diffuse proliferative Lupus Glomerulonephritis treated with sequential oral cyclophosphamide and azathioprine. Arthritis Rheum 2002;46:1003-1013.

62. Mok CC. Prognostic factors in Lupus Nephritis. Lupus 2005;14:39-44.

63. Flower C, Hennis A, Hambleton IR, Nicholson G. Lupus Nephritis in afro-caribbean population: Renal indices and clinical outcome. Lupus 2006;15:689-694.

64. Gibson K, Gipson D, Massengill S, Dooley MA, Primack W, Ferris M, et al. Predictors of relapse and end stage kidney disease in proliferative lupus nephritis: Focus on children, adolescents and Young adults. Clin J Am Soc Nephrol 2009;4:1962-1967.

65. Moon SJ, Kwok SK, Ju JH, Park KS, Park SH, Cho CS, et al. Predictors of chronic kidney disease in Korean patients with lupus nephritis. J Rheumatol 2011;38:2588-2597. 
66. So MW, Koo SB, Kim YG, Lee ChK, Yoo B. Predictive value of remission status after 6-month induction therapy in patients with proliferative Lupus Nephritis. A retrospective analysis. Clin Rheumatol 2011;30:1399-1405.

67. Levey AS, Lan SP, Corwin HL, Kasinath BS, Lachin J, Nielson EG, et al. Progression and remission of renal disease in Lupus Nephritis collaborative study: Results of treatment with prednisone and short-term oral cyclophosphamide. Ann Int Med 1992;116:114-123.

68. Fraenkel L, Hayslett JP, Esdaile J. Response to treatment as predictor of long-term outcome in patients with Lupus Nephritis. J Rheumatol 1994;21:2052-2057.

69. Houssiau F Vasconcelos C, D 'Cruz D, Sebastiani GD, De Ramón-Garrido ER, Danielli MC, et al. Early response to immunosuppressive therapy predicts good renal outcome in Lupus Nephritis. Lessons from long-term follow-up of patients in Eurolupus Nephritis Trial. Arthritis Reum 2004;50:3934-3940.

70. The American College of Rheumatology Response Criteria for Proliferative and Membranous Renal Disease in Systemic Lupus Erythematosus Clinical Trials. Arthritis Rheum 2006;54:421-432.

71. Austin III HA, Klipel JH, Ballow J, Le Riche N, Steimberg A, Plotz P, et al. Therapy for Lupus Nephritis Controlled Trial of prednisone and cytotoxic drugs. N Eng J Med 1986;314:614-619.

72. Lai K, Sydney C, Mok CC. Treartment for Lupus Nephritis. A Revisit. Nephrol 2005;10:180-188.

73. Illei G, Austin III H, Crane M, Collins L, Gourley M, Yarboro Ch, et al. Combination therapy with pulse cyclophosphamide plus pulse methylprednisolone improve long-term renal outcome without adding toxicity in patients with Lupus Nephritis. Ann Int Med 2001;135:248-257.

74. Houssiau F Vasconcelos C, D Cruz D, Sebastiani GD, De Ramón-Garrido ER, Danielli MC, et al. The 10-year Follow-up data of the Eurolupus Nephritis Trial comparing low-dose and high-dose intravenous cyclophosphamide. Ann Rheum Dis 2010;69:61-64.

75. Appel GB, Contreras G, Dooley MA, Ginzler E, Isenberg D, Jayne D, et al. Mycophenolate Mofetil versus cyclophosphamide for induction treatment of Lupus Nephritis. J Am Soc Nephrol 2009;20:1103-1112.

76. Ginzler EM, Dooley MA, Arnow C, Kim M, Buyon J, Merrill JT, et al. Mycophenolate Mofetil or intravenous cyclophosphamide for Lupus Nephritis. N Eng J Med 2005;353:2219-2228.

77. Chan TM, Li FK, Tang CS, Wong R, Fang G, Ji Y, et al. Efficacy of Micophenolate Mofetil in patients with Diffuse Proliferative Lupus Nephritis. Hong Kong Guangzhou Nephrology Study Group. N Eng J Med 2000;343:1156-1162.

78. Ong M, Hooi LS, Tim TO, Go BL, Ahmad G, Ghazalli R. et al. Randomized controlled trial of pulse intravenous cyclophosphamide versus mycophenolate mofetil in the induction therapy of proliferative Lupus Nephritis. Nephrol 2005;10:504-510.

79. Touma Z, Gladman D, Urowitz M, Beyene J, Ulerik E, Shah P. Mycophenolate mofetil for induction treatment of lupus nephritis: A systematic review and meta-analysis. J Rheumatol 2011;38:69-78.

80. Henderson L, Masson P, Craig J, Roberts M, Flanc R, Strippoli G. Induction and Maintenance Treatment of proliferative Lupus Nephritis: A meta-analysis of randomized controlled trials. Am J Kidney Dis 2013;61:74-87.

81. Walsch M, Solomons N, Lisk L, Jayne D. Mycophenolate mofetil or intravenous cyclophosphamide for lupus nephritis with por kidney function: A subgroup Analysis of ASPREVA lupus manegement study. Am J Kidney Dis 2013;61:710-715.

82. Tang Z, Yang G, Yussheng Y, Jinquan W, Weixin H, Zeng C, et al. Effect of mycophenolate mofetil for patients with crescentic lupus nephritis. Neprol 2008;13:702-705.

83. Wang J, Hu W, Zhang H, Chen H, Zeng C, Liu Z, et al. Induction therapies for class IV Lupus Nephritis with non-inflammatory necrotizing vasculopathy: Mycophenolate mofetil or intravenous cyclophosphamide. Lupus 2007;16:707-712.

84. Marimoto S, Watanabe T, Lee S, Amano H, Kammaro Y, Ohsawa I, et al. Improvement of rapidly progressive lupus nephritis aasociated MPO-ANCA with tacrolimus. Mod Rheumatol 2010;20:291-294.

85. Yap D, Ma M, Mok M, Tang C, Chan TM. Long-term data on corticosteroids and Mycophenolate mofetil treatment in Lupus Nephritis. Rheumatol 2013;52:480-486. 
86. Contreras G, Pardo V, Leclerq B, Lenz O, Tozman E, O`nan P, et al. Sequential therapies for proliferative lupus nephritis. N Eng J Med 2004:350:971-980.

87. Dooley MA, Jayne D, Ginzler E, Isenberg D, Olsen N, Wofsky D, et al. Mycophenolate versus azathioprine an maintenance therapy for Lupus Nephritis. N Eng J Med 2011;365:1886-1895.

88. Houssiau FA, D 'Cruz D, Sangle S, Remy P, Vasconcelos C, Petrovic R. Azathioprine versus mycophenolate mofetil for long-term immunosuppression in Lupus Nephritis: Results from the MAINTAIN nephritis trial. Ann Rheum Dis 2010;69:2083-2089.

89. Chan TM, Tse KC, Tang CS, Mok MY, Li FK. Long-term study of mycophenolate mofetil as continuous induction and maintenance treatment for diffuse proliferative Lupus Nephritis. J Am Soc Nephrol 2005;16:1076-1084.

90. Feng Li, Deng J, Huo DM, Wu QY, Liao YH. Mycophenolate mofetil versus azathioprine as maintenance therapy for Lupus Nephritis: A meta-analysis. Nephrol 2013;18:104-110.

91. Rovin B, Furie R, Latinis K, Looney J, Fervenza F, Sánchez-Guerrero J, et al. Efficacy and safety of rituximab in patients with active proliferative Lupus Nephritis, Arthritis Rheum 2012;64:1215-1226.

92. Díaz-Lagares C, Croca S, Sangle S, Vital E, Catapano F, Martínez-Berriotxa A, et al. Efficacy of Rituximab in 164 patients with Biopsy proven lupus nephritis: Pooled data from european cohorts. Autoimm Rev 2012;11:357-364.

93 Jondasttir T, Gunnarson I, Mourao A, Lu T, Van Vollenhoven R, Isenberg D. Clinical improvement in proliferative versus membranous lupus nephritis following B cell depletion: pooled data from two cohorts. Rheumatol 2010,49:1502-1504.

94. Terier B, Amoura Z, Ravaud P, Hachulla E, Jovenne R, Combe B, et al. Safety and efficacy of rituximab in Systemic Lupus Erythematosus. Results from 136 patients from the french autoimmunity and rituximab registry. Arthritis Rheum 2010;62: 2458-2466.

95. García-Carrasco M, Mendoza-Pinto C, Sandoval-Cruz M, Soto-Vega E, Beltrán-Castillo A, Jiménez-Hernández M, et al. Anti-CD20 therapy in patients with refractory systemic lupus erythematosus: A longitudinal analysis of 52 hispanics. Lupus 2010;19:213-219.

96. Melander C, Sallee M, Trolliet P, Candon S, Belenfant X, Daugas E, et al. Rituximab in severe lupus nephritis: Early B cell depletion affects, long-term renal outcome. Clin J Am Soc Nephrol 2009;4:579-587.

97. Moroni G, Raffiotta F, Trezi B, Giglio E, Mezzina N, Delpapa N, et al. Rituximab versus Mycophenolate Mofetil versus Cyclophosphamide pulses for induction therapy of active lupus nephritis: A clinical observational study. Rheumatol 2014;53:1570-1577.

98. Condon M, Ashby D, Peeper R, Cook H, Levy J, Griffith M, et al. Prospective observational single-center cohort study to evaluate the effectiveness of treating Lupus Nephritis with rituximab and mycophenolate but no oral steroids. Ann Rheum Dis 2013;72:1280-1286.

99. Li E, Tam LS, Zhu T, Li M, Kwok K, Li T. Is combination rituximab with cyclophosphamide better tan rituximab alone in the treatment of Lupus Nephritis? Rheumatol 2009;48:892-898.

100. Ramos-Casals M, SDoto MJ, Cuadrado MJ, Khamashta M. Rituximab in Systemic Lupus Erythematosus: A systematic review of off-label use in 188 cases. Lupus 2009;18:767-776.

101. Ogawa H, Kameda H, Nagasawa H, Sekiguchy N, Takei H, Tzuzaka K, et al. Prospective study of low-dose cyclosporine in patients with refractory Lupus Nephritis. Mod Rheumatol 2007;17:92-97.

102. Fesser BJ, Alarcón GS, Mc Gwin F Jr, Roseman J, Bastian HM, Friedman AW, et al. For the LUMINA Study Group. Systemic Lupus Erythematosus in three etnic groups. XVI. Association of hydroxicholoquine use with reduced risk of damage accrual. Arthritis Rheum 2005;52:1473-1480.

103. Pons-Estel GJ, Alarcón GS, McGwin G Jr, Danila MI, Zhang J, Bastian HM, et al. For the LUMINA Study Group. Protective effect of hydroxichloroquine of renal damage in patients with Lupus Nephritis: LXV. Data from, a multietnic US cohort. Arthritis Rheum 2009; 61:863-868. 